Cahiers $d u$ MONDE RUSSE

\section{Cahiers du monde russe}

Russie - Empire russe - Union soviétique et États indépendants

$50 / 2-3 \mid 2009$

L'Europe orientale, 1650-1730. Crises, conflits et renouveau

\title{
Literary Scholarship in Late Imperial RussiaAndy BYFORD
}

, Londres : Legenda, Modern Humanities Research Association and Maney Publishing, 2007, 188 p.

\section{Catherine Depretto}

\section{(2) OpenEdition}

\section{Journals}

Édition électronique

URL : https://journals.openedition.org/monderusse/9753

DOI : $10.4000 /$ monderusse. 9753

ISSN : $1777-5388$

Éditeur

Éditions de l'EHESS

Édition imprimée

Date de publication : 15 septembre 2009

ISBN : 978-2-7132-2260-3

ISSN : $1252-6576$

Référence électronique

Catherine Depretto, "Literary Scholarship in Late Imperial RussiaAndy BYFORD », Cahiers du monde russe [En ligne], 50/2-3 | 2009, mis en ligne le 14 janvier 2013, consulté le 03 septembre 2022. URL http://journals.openedition.org/monderusse/9753; DOI : https://doi.org/10.4000/monderusse.9753

Ce document a été généré automatiquement le 3 septembre 2022

Tous droits réservés 


\section{Literary Scholarship in Late Imperial RussiaAndy BYFORD}

, Londres : Legenda, Modern Humanities Research Association and Maney Publishing, 2007, 188 p.

\section{Catherine Depretto}

\section{Andy BYFORD, Literary Scholarship in Late Imperial Russia, Rituals of Academic Institutionalisation, Londres : Legenda, Modern Humanities Research Association and Maney Publishing, 2007, 188 p.}

1 Si les grands noms de la critique littéraire russe $a u x^{e}$, à commencer par les formalistes, sont assez célèbres, on connait mal, en revanche, exception faite d'Aleksandr Veselovskij et d'Aleksandr Potebnja, leurs prédécesseurs immédiats, les savants qui, au tournant des $\mathrm{XIX}^{\mathrm{e}}$ et $\mathrm{xx}^{\mathrm{e}}$ siècles, ont contribué à l'émergence d'une science de la littérature. Cet ouvrage d'histoire sociale ou de sociologie historique s'emploie à combler cette lacune. Son angle d'approche n'est pas l'étude immanente des textes concernés par la période, mais la professionnalisation de la discipline, autrement dit l'étude de la façon dont les acteurs sociaux (ici les spécialistes de littérature ${ }^{7}$ ) ont construit leur identité professionnelle, à travers un certain nombre d'études, mais aussi par le biais de célébrations, discours d'intronisation, séminaires, biographies, etc. L'auteur, citant Cassirer et Durkheim, se réfère principalement aux travaux de Pierre Bourdieu et reprend ses catégories: "principes de vision et de division du monde social», "rites d'institution », « habitus », « champ ».

2 Après une introduction qui définit le sujet et énonce les principes de l'étude, l'ouvrage comporte quatre chapitres. Le premier retrace l'histoire de l'enseignement universitaire en Russie à partir de sa fondation et souligne le rôle de l'Académie des sciences, absorbant en 1841 l'Académie russe, fondée par Catherine II. Ce rappel historique est centré sur les études littéraires dont l'essor coïncide, selon l'auteur, avec les efforts de constitution d'une nation russe moderne, c'est-à-dire avec la volonté d'affirmer 
l'existence d'une culture nationale spécifique, ce dont témoignent l'apparition des chaires de langue et littérature russes, l'intérêt pour le folklore et la poésie populaire russes, l'importance des débats autour de la narodnost'. Ces tendances se retrouvent dans les ouvrages de savants comme Fedor Buslaev, Aleksandr Afanas'ev, Orest Miller, qui, tout en aspirant à faire œuvre scientifique, veulent aussi travailler à l'éducation de la société dans son ensemble (en particulier Aleksandr Pypin).

Le deuxième chapitre analyse la façon dont les anniversaires, commémorations sont pour la communauté scientifique l'occasion de rites d'institutions. On sait qu'à partir de la seconde moitié du $\mathrm{XIX}^{\mathrm{e}}$ et en particulier du tournant des $\mathrm{XIX}^{\mathrm{e}}$ et $\mathrm{XX}^{\mathrm{e}}$ siècles, les célébrations de toute sorte, celles des classiques de la littérature au premier chef, ont joué un rôle important dans l'affirmation de la conscience nationale. L'intérêt de ce chapitre est qu'il ne se limite pas aux capitales, mais s'intéresse à des institutions moins connues de province (Société historique et philologique de l'université d'Odessa 1904, de Har'kov 1902). Il s'arrête également sur deux institutions particulières, le département de langue russe et de littérature de l'Académie des sciences (Otdelenie russkogo jazyka i slovesnosti, ORJAS) de Saint-Pétersbourg et ce qui aspire à jouer à Moscou un rôle équivalent, la Société des amoureux de la littérature russe (Obščestvo ljubitelej russkoj slovesnosti, OLRS). L'étude de ces célébrations est le moyen de voir comment la communauté littéraire savante s'autodéfinit, comment elle cherche à se situer par rapport à l'État comme par rapport à la société. Par-delà des situations et aspirations spécifiques, l'auteur met en évidence des éléments rhétoriques communs qui visent à assurer aux savants une position intermédiaire entre autorité scientifique supérieure et liens avec la littérature et la communauté non scientifique dans son ensemble, ainsi qu'une attitude de loyauté à l'égard de la monarchie, associée à la volonté d'être le porte-parole de la société et de servir la cause de son éducation.

4 Le troisième chapitre s'intéresse aux biographies le plus souvent hagiographiques de plusieurs figures importantes de la discipline, symboles de trois générations successives, principalement Jakov Grot, Fedor Buslaev, Aleksandr Veselovskij, Aleksandr Potebnja et Dmitrij Ovsjaniko-Kulikovskij. L'analyse comparée de ces biographies, de nature très différente, permet néanmoins à l'auteur de mettre en évidence une image mythique du spécialiste de littérature idéal qui se caractériserait par les traits suivants: vocation précoce, révélation de la science pendant les études universitaires, suivies le plus souvent d'un voyage à l'étranger, sans que faiblisse le sentiment patriotique, recherche de la vérité et sentiment fort d'obligation morale, qui pousse à résister à la tentation de la tour d'ivoire académique, conjonction d'un savant et d'un enseignant (p. 117). Le quatrième et dernier chapitre analyse des textes programmatiques, leçons inaugurales, préfaces, introductions méthodologiques, comme des entreprises d'érudition (dictionnaires, encyclopédies, histoire de la littérature), toujours sous l'angle de la position dans le champ des savants considérés.

5 La conclusion de Byford (qui est en même temps l'objectif déclaré du livre) est de montrer que ces spécialistes de littérature, traités souvent avec condescendance aussi bien en Russie qu'à l'étranger, ont cherché à affirmer la légitimité de leur discipline et ont largement contribué à l'institutionnalisation d'une science de la littérature en Russie, en se situant face à cinq réalités : la science européenne, l'État, la société, la littérature et l'enseignement.

6 Cet ouvrage constitue donc une sorte de réhabilitation de ces « courants académiques ", présentés, selon l'auteur, de manière trop simplificatrice, comme souvent éclipsés par la 
critique engagée de l'époque et qui peuvent être considérés comme « les structures de base » (root structure) des formalistes. L'ouvrage est très bien présenté, illustré par une belle iconographie, complété d'une bibliographie et d'un index.

7 Il s'agit d'un travail érudit et brillant qui s'inscrit dans le courant des études récentes de sociologie historique, consacrées aux élites intellectuelles russes, et qui renouvelle l'approche de l'histoire des études littéraires en Russie. Néanmoins, on peut se demander si l'auteur n'exagère pas la méconnaissance dont seraient victimes les courants académiques de la critique littéraire. Les mérites de Buslaev, Pypin, Veselovskij, Potebnja, Vengerov sont bien connus et la dette des mouvements suivants à leur égard est également étudiée. De la même façon, la volonté de mettre en évidence les processus d'institutionnalisation de la discipline pousse peut-être Byford à mettre sur le même plan des phénomènes de nature différente et à surestimer les préoccupations méthodologiques de certains représentants de ces courants. Ainsi l'auteur affirme prendre le contre-pied de l'idée selon laquelle le tournant des $\mathrm{XIX}^{\mathrm{e}}$-Xx ${ }^{\mathrm{e}}$ siècles serait caractérisé par une crise des études littéraires. Cette idée qu'on peut trouver sans doute dans certains travaux de la période soviétique présentant unilatéralement les dernières années de l'ancien régime comme des années de crise ${ }^{8}$ n'est en tout cas pas celle qui a cours dans les travaux universitaires les plus récents. On regrette que, comme souvent dans les travaux anglo-saxons, l'historiographie slavisante de langue française, de même que les travaux européens en général, soient très peu pris en considération.

8 Ces quelques remarques n'enlèvent rien à la qualité de cet ouvrage, le premier à aborder les études littéraires en Russie sous l'angle des processus d'institutionnalisation et qui, à ce titre, constitue une étude véritablement pionnière.

\section{NOTES}

7. C'est ainsi que nous rendons ici l'anglais literary scholar, traduit plus haut par savant.

8. Les formalistes russes ont également eu tendance à souligner la faiblesse méthodologique de leurs prédécesseurs alors même qu'ils leur devaient beaucoup, $\mathrm{cf}$. l'importance du séminaire de Semen Vengerov sur Puškin et du séminaire roman et germanique de Dmitrij Petrov à l'université de Saint-Pétersbourg dans les années 1910. 